\title{
Cystatin C as the Predictor of Chronic Kidney Disease in Patients with Comorbid Pathology
}

\author{
IT Murkamilov*1,2, VV Fomin ${ }^{3}$, KA Aitbaev ${ }^{4}$, Kinvanlun IG ${ }^{2}$ and Zh A Murkamilova ${ }^{5}$ \\ ${ }^{1}$ Department of Faculty Therapy, Kyrgyz State Medical Academy, Kyrgyzstan \\ ${ }^{2}$ Head of the Department of Faculty Therapy, Federal State Autonomous Educational Institution of Higher Education "First Moscow State Medical University, \\ Russia
}

${ }^{3}$ Head lab Pathological Physiology Institute of Molecular Biology and Medicine, Specialists in Chronic Kidney Disease of Kyrgyzstan, Russia

${ }^{4}$ Teacher, Scientific Research Institute of Molecular Biology and Medicine, Kyrgyzstan

${ }^{5}$ Physician, Family Medicine Center, Kyrgyzstan

*Corresponding author: IT Murkamilov, Department of Faculty Therapy, Specialists

in Chronic Kidney Disease of Kyrgyzstan, Kyrgyzstan, Russia.

Received Date: July 08, 2019

Published Date: July 30, 2019

\begin{abstract}
Summary
Purpose of the study: To study the content of cystatin C and to identify the factors reducing the glomerular filtration rate in patients with comorbid pathology.

Materials and methods: 383 patients with comorbid pathology aged from 25 to 88 years (mean age $58.8 \pm 12.0$ years) were examined, of whom $51.4 \%$ were men and $48.6 \%$ women. In the structure of comorbid pathology, arterial hypertension $(\mathrm{AH})$ was observed in $76.5 \%$, coronary heart disease (CHD) in $48.8 \%$, diabetes mellitus (DM) type2 in $29.7 \%$, obesity in $47,5 \%$ and chronic obstructive pulmonary disease (COPD) in $28.9 \%$ of patients. The examined patients were divided into 4 groups: $1^{\text {st }}$ persons with hypertension+type 2 diabetes $(n=99) ; 2^{\text {nd }}-A H+C H D(n=138) ; 3^{\text {rd }}-A H$ + COPD $(n=102)$ and the $4^{\text {th }}$ group patients with severe comorbid pathology, i.e. AH + DM + CHD + COPD $(n=44)$. All patients were studied for lipid spectrum parameters [total cholesterol (cholesterol), high-density lipoprotein cholesterol (HDL cholesterol), low-density lipoprotein cholesterol (LDL cholesterol), triglycerides (TG)] and cystatin C of blood plasma. The glomerular filtration rate (GFR) was calculated according to the formula F.J. Hoek. The severity of renal dysfunction was assessed according to the recommendation of KDIGO (Kidney Disease: Improving Global Outcomes) 2012. The analysis of the prevalence of reduced GFR in patients with comorbid pathology was also carried out.
\end{abstract}

Results: The presence of reduced GFR gradations of C2 and C3 "A" was detected in $46.7 \%$ and $24.8 \%$ of the examined individuals, respectively. Severe reduction in GFR was observed in $11.7 \%$ of patients. Sharply reduced GFR (C4 gradation) was significantly more frequently registered in women than in men $(10.7 \%$ versus $5.0 \% ; \mathrm{p}<0.05)$. In the examined cohort, persons of middle and old age made up $36.8 \%$ and $42.2 \%$, respectively. In patients with comorbid pathology, cases of hypercholesterolemia (41.7\%), hypertriglyceridemia (45.4\%) and elevated levels of LDL cholesterol (46.9\%) were frequently detected, and in the $4^{\text {th }}$ group, the mean age and body mass index (BMI) were significantly higher, and cholesterol levels of HDL and GFR were significantly lower compared with other groups. In the 1st group (AH+DM type2), systolic blood pressure (SBP), total cholesterol and cystatin $\mathrm{C}$ plasma levels were significantly higher than in the $\mathrm{AH}+\mathrm{CHD}, \mathrm{AH}+\mathrm{COPD}$ groups and patients with severe comorbid pathologies. Higher concentrations of LDL cholesterol were observed in patients with hypertension and COPD. In the $1^{\text {st }}$ group of patients, the GFR value closely correlated with age $(\mathrm{r}=-0.21 ; \mathrm{p}=0.03)$, the level of SBP $(\mathrm{r}=-0.34 ; \mathrm{p}=0.03)$, and concentration cystatin $\mathrm{C}$ of blood plasma $(\mathrm{r}=-0.87 ; \mathrm{p}=0.01)$. In the $2^{\text {nd }}$ subgroup, the GFR index was associated with the level of the SBP ( $r=-0.32$; $p=0.03)$ and the concentration of cystatin $C$ in the blood plasma $(r=-0.86$; $p=0.03)$. In patients with AH, COPD negative association of GFR was recorded with age ( $r=-0.44 ; \mathrm{p}=0.03)$, heart rate $(\mathrm{r}=-0.36$; $\mathrm{p}=0.03)$, total cholesterol concentration ( $r=-0.20 ; p=0.04)$, HDL cholesterol levels ( $r=-0.27 ; p=0.03)$, LDL cholesterol levels ( $r=-0.27 ; p=0.03)$, as well as cystatin C ( $r=-0.89$; $\mathrm{p}=0.01)$. In the $4^{\text {th }}$ subgroup, the magnitude of GFR was significantly correlated with the level of SAP $(\mathrm{r}=-0.33$; $\mathrm{p}=0.03)$ and the concentration of cystatin $\mathrm{C}$ of blood plasma ( $\mathrm{r}=-0.86 ; \mathrm{p}=0.01)$.

Findings: In patients with comorbid pathology, the frequency of reduction in glomerular filtration rate of the C2 gradation was $46.7 \%$, and the C3 "A" gradation was $24.8 \%$. Severe decline in renal function was observed in $11.7 \%$ of patients, and it was more often detected in women. In comorbid pathology, a decrease in renal function is associated with the presence of hypercholesterolemia, hypertriglyceridemia, and an elevated level of cholesterol of low-density lipoprotein. In individuals with comorbid pathology, the incidence and severity of chronic kidney disease are influenced by age, body mass index, systolic blood pressure, total cholesterol and cystatin C concentrations of blood plasma.

Keywords: Cystatin C; Glomerular filtration rate; Chronic kidney disease; Comorbid pathology 


\section{Introduction}

Chronic kidney disease (CKD) is still one of the most significant medical and social problems of our time [1,2]. In Kyrgyzstan, by the beginning of April 2019, the number of patients receiving program hemodialysis reached more than 1,400 people. At the same time, persons with comorbid pathology, type2 diabetes mellitus (DM), hypertension and chronic glomerulonephritis, predominate among them. The definition of comorbidity was first given by the American epidemiologist A.R. Feinstein in 1970 [3]. Over the past five years, the number of studies on comorbid pathology, especially comorbidity for cardiovascular diseases (CVD), has increased significantly [4-7]. A distinctive feature of CKD is also a high frequency of comorbidity, which does not depend on age and gender. Numerous studies have found that in patients with CKD, cardiovascular risks are several times higher than in the general population of people, and to a large extent determine the survival rate of this category of persons $[8,9]$. Comorbidity in CKD leads to a mutual influence on the course of diseases, the nature and severity of complications, often makes it difficult to diagnose and determines the characteristics of nephroprotective therapy $[1,2]$. In recent years, there is a need for a comprehensive study of the pathogenetic mechanisms of the development of CKD, including in comorbid patients. Purpose of the study. To study the content of cystatin $\mathrm{C}$ and to identify the factors reducing the glomerular filtration rate in patients with comorbid pathology.

\section{Materials and Methods}

The study included 383 patients with comorbid diseases. Among the patients there were $51.4 \%$ of men and $48.6 \%$ of women aged from 25 to 88 years (mean age- $58.8 \pm 12.0$ years). In the structure of comorbid pathology, arterial hypertension (AH) were 293 (76.5\%) cases, coronary heart disease (CHD)-187 (48.8\%), diabetes mellitus (DM) type2-114 (29.7\%), obesity-182 (47.5\%) and chronic obstructive pulmonary disease (COPD)-111 (29.0\%). The present study, in which patients with comorbid pathology took part, was approved by the Ethics Committee of KSMA named after I.K. Akhunbaev (Bishkek) and informed consent of the patients to the study was obtained. For the comorbid pathology, they took the presence in the patient of two or more diseases pathogenically interrelated with each other, coinciding in time or being a complication resulting from the underlying disease or its treatment.

Patients with cirrhosis of the liver and the 5 "D" stage of CKD, a severe form of thyrotoxicosis, paraneoplastic processes, pregnant women and people taking high doses of corticosteroids were not included in this study. Blood pressure (BP, $\mathrm{mm} \mathrm{Hg}$ ), height ( $\mathrm{cm}$ ), and weight $(\mathrm{kg})$ were measured in all patients and the body mass index (BMI, $\mathrm{kg} / \mathrm{m}^{2}$ ) was determined. For the criterion of hypertension took the blood pressure level equal to or more than $140 / 90 \mathrm{mmHg}$., or a lower level of blood pressure on the background of antihypertensive therapy. A BMI value $>29.9 \mathrm{~kg} / \mathrm{m}^{2}$ was considered as obesity. Biochemical blood tests included: determination of total cholesterol (cholesterol), high-density lipoprotein cholesterol (HDL cholesterol), low-density lipoprotein cholesterol (LDL cholesterol) and serum triglycerides (TG). Additionally, in all patients, the concentration of cystatin C (mg/l) of serum was investigated using the immunoturbidimetric method.

Hypercholesterolemia was classified with total cholesterol content of more than $5.01 \mathrm{mmol} / \mathrm{l}$, hypertriglyceridemia-TG more than $1.7 \mathrm{mmol} / \mathrm{l}$, high LDL - more than $3.0 \mathrm{mmol} / \mathrm{l}$. The renal function was assessed using the GFR, the calculation of which was carried out according to the formula F.J. Hoek and authors, proposed in 2003. (80.35/CystatinC-4.32=GFR) [10]. Clinical forms of the disease in the examined patients, such as the stable course of CHD, type 2 diabetes and COPD were exposed on the basis of the submitted medical documentation. According to the WHO recommendations, the examined patients were divided into young age (18-29 years old), mature age (30-44 years), average age (4559 years), elderly and senile (75-89) years [11]. For a comparative assessment of kidney function, groups with various combinations of significant comorbid pathologies were identified: AH + type $2 \mathrm{DM}$ ( $n=99), A H+$ stable types of CHD(n=138), AH+COPD ( $=102)$ and $A H+D M+C H D+C O P D(n=44)$. Statistical processing of the data was performed using the statistical software package Statistical 10.0. Data are presented as medians (25-75 percentiles). Differences between groups were evaluated using Mann-Whitney criteria (in the case of non-parametric distribution of the trait) and Student's t-test (if there are signs with a normal distribution). Correlation analysis was performed by the Pearson criterion (for variables with a normal distribution) and the Spearman coefficient (for variables with a non-parametric distribution). A critical level of statistical significance was taken 0.05 .

\section{Results}

(Table 1) presents the clinical and demographic indicators of the examined patients. Thus, in the total sample $(\mathrm{n}=383)$, patients with C2 (46.7\%) and C3 "A" (24.8\%) were dominated by gradations of renal dysfunction. Severe decline in renal function was observed in $11.7 \%$ of patients. However, optimal renal function (C1) was detected in only $7.5 \%$ of the examined individuals with comorbid pathology (Table 1). A comparative analysis of the frequency of occurrence of reduced renal function showed the following results. Thus, the proportion of patients with $\mathrm{C} 1$ gradation of renal dysfunction was significantly less in men compared with women (4.0\% vs. $11.2 \%$; $\mathrm{p}<0.05$ ).

The frequency of occurrence of C2, C3 " $A$ ", C3 "B" and C5 gradations of impaired renal function in men and women did not differ significantly ( $p>0.05)$. Sharply reduced calculated GFR, i.e. $\mathrm{C} 4$ gradation was significantly more frequently recorded in women than in men $(10.7 \%$ vs. $5.0 \%$; $\mathrm{p}<0.05)$. Analysis of the age structure of the patients examined by us showed that the proportion of middle-aged and elderly people was the most numerous $(36.8 \%$ and $42.2 \%$, respectively). Among men ( $n=197)$, patients in adulthood and old age were significantly higher than among women ( $p>0.05)$. In addition, patients with comorbid pathology often identified laboratory predictors of CKD progression, 
such as hypercholesterolemia (41.7\%), hypertriglyceridemia (45.4\%), and elevated LDL-C (46.9\%). It is important to note that hypercholesterolemia was significantly more frequent among women $(\mathrm{n}=186)(51.0 \%$ vs. $32.9 \%$; $\mathrm{p}<0.05)$ than among men (Table 1).

Table 1: Clinical and Demographic Characteristics of the Examined Patients.

\begin{tabular}{|c|c|c|c|}
\hline $\begin{array}{c}\text { Category of Calculated GFR, ml/min KDIGO (Kidney } \\
\text { Disease: Improving Global Outcomes) }\end{array}$ & Total $n=383$ & Men $n=197$ & Women $\mathrm{n}=186$ \\
\hline C1, n (\%) & $29(7,57)$ & $8(4,0)^{*}$ & $21(11,2)$ \\
\hline C2, n (\%) & $179(46,7)$ & $95(48,2)$ & $84(45,1)$ \\
\hline $\mathrm{C} 3 \ll \mathrm{A} », \mathrm{n}(\%)$ & $95(24,8)$ & $54(27,4)$ & $41(22,0)$ \\
\hline $\mathrm{C} 3$ «B», n (\%) & $35(9,1)$ & $20(10,1)$ & $15(8,0)$ \\
\hline $\mathrm{C} 4, \mathrm{n}(\%)$ & $30(7,8)$ & $10(5,0)$ & $20(10,7)^{*}$ \\
\hline $\mathrm{C} 5, \mathrm{n}(\%)$ & $15(3,9)$ & $10(5,0)$ & $5(2,6)$ \\
\hline Young age, n (\%) & $9(2,3)$ & $8(4,0)$ & $1(0,5)$ \\
\hline Mature age, n (\%) & $36(9,3)$ & $23(11,6)^{*}$ & $13(6,9)$ \\
\hline Meanage, n (\%) & $141(36,8)$ & $77(39,0)$ & $64(34,4)$ \\
\hline Older age, n (\%) & $162(42,2)$ & $74(37,5)^{*}$ & $88(47,3)$ \\
\hline Senile age, n (\%) & $35(9,1)$ & $15(7,6)$ & $20(10,7)$ \\
\hline Hypercholesterolemia, n (\%) & $160(41,7 \%)$ & $65(32,9 \%)$ & $95(51,0 \%)^{*}$ \\
\hline Hypertriglyceridemia, n (\%) & $174(45,4 \%)$ & $88(44,6 \%)$ & $86(46,2 \%)$ \\
\hline Increased level of LDL cholesterol, n (\%) & $180(46,9 \%)$ & $94(47,7 \%)$ & $86(46,2 \%)$ \\
\hline
\end{tabular}

Note: EGFR-estimated glomerular filtration rate; LDL - low density lipoprotein cholesterol; $\mathrm{n}$ is the number of observations; $\%$ is the absolute number of patients; ${ }^{*}-\mathrm{p}<0.05$.

As already noted, to assess renal function between different types of comorbid pathology, the sample of patients under study was divided into groups, the comparative clinical and laboratory characteristics of which are presented in Table 2. As follows from it, in patients with a pronounced comorbid state (group 4), the mean age and BMI indices were significantly higher $(\mathrm{p}<0.05)$, and the level of LDL cholesterol and GFR were significantly lower $(p<0.05)$ compared to other groups. In the $1^{\text {st }}$ group (AH+DM type 2$)$, the values of systolic blood pressure, total cholesterol and cystatin $\mathrm{C}$ of serum were significantly higher $(\mathrm{p}<0.05)$ in comparison with other groups (AH+COPD, $\mathrm{AH}+\mathrm{CHD}$ and $\mathrm{AH}+\mathrm{DM}+\mathrm{CHD}+\mathrm{COPD})$ (Table 2).

Comparatively higher concentrations of LDL cholesterol were observed in patients with hypertension and COPD. As for the serum TG level, no intergroup differences were found for this indicator. There was also no significant difference between the groups in terms of diastolic blood pressure and heart rate (Table 2).

Table 2: Comparative Clinical and Laboratory Characteristics of the Examined Subgroups..

\begin{tabular}{|c|c|c|c|c|}
\hline Parameters & $\mathbf{1}^{\text {st }}$ Subgroup AH + DM, n=99 & $\begin{array}{c}2^{\text {nd }} \text { Subgroup AH+CHD, } \\
\mathrm{n}=138\end{array}$ & $\begin{array}{c}3^{\text {rd }} \text { Subgroup AH+COPD, } \\
\mathrm{n}=102\end{array}$ & $\begin{array}{c}4^{\text {th }} \text { Subgroup } \\
\text { AH+DM+CHD+COPD, } \mathrm{n}=44\end{array}$ \\
\hline Age, years & $59,7 \pm 9,3$ & $63,1 \pm 9,1$ & $60,9 \pm 12,1$ & $64,1 \pm 7,6^{*}$ \\
\hline BMI, $\mathrm{kg} / \mathrm{m} 2$ & $30,4 \pm 6,0$ & $28,5 \pm 5,6$ & $27,9 \pm 6,6$ & $142 \pm 24$ \\
\hline SBP, mm Hg & $144 \pm 25^{* * *}$ & $140 \pm 23$ & $128 \pm 15$ & $85 \pm 11$ \\
\hline DBP, mm Hg & $87 \pm 11$ & $85 \pm 10$ & $82 \pm 8$ & $78 \pm 13$ \\
\hline HR, beats/min & $80 \pm 11$ & $78 \pm 14$ & $81 \pm 14$ & $4,63 \pm 1,36$ \\
\hline Cholesterol, mmol/l & $5,15 \pm 1,82^{* * * *}$ & $4,69 \pm 1,34$ & $4,77 \pm 1,29$ & $1,70(1,21 ; 2,33)$ \\
\hline TG, mmol/l & $1,76(1,26 ; 2,51)$ & $1,52(1,01 ; 2,27)$ & $1,33(0,95 ; 1,70)$ & $1,05 \pm 0,28^{* * *}$ \\
\hline HDL cholesterol, mmol/l & $1,11 \pm 0,31$ & $1,09 \pm 0,29$ & $1,16 \pm 0,32$ & $2,91(2,33 ; 3,69)$ \\
\hline LDL cholesterol, mmol/l & $3,13(2,47 ; 3,95)$ & $2,86(2,18 ; 3,58)$ & $3,22^{* *}(2,36 ; 3,97)$ & $1,56(1,23 ; 2,39)$ \\
\hline Cystatin C, mg/l & $1,88^{* * *}(1,05 ; 2,46)$ & $1,34(1,11 ; 1,91)$ & $1,22(1,09 ; 1,39)$ & $48,1^{* * *}(32,0 ; 61,0)$ \\
\hline EGFR, ml/min & $52,2(29,2 ; 72,2)$ & $56,0(38,1 ; 68,0)$ & $61,5(53,4 ; 69,3)$ & 4 \\
\hline
\end{tabular}

Note: AH-arterial hypertension; DM-Diabetes mellitus; CHDcoronary heart disease; COPD-chronic obstructive pulmonary disease; BMI-body mass index; SBP-systolic blood pressure; DBP-diastolic blood pressure; HR-heart rate; Cholesterol-total cholesterol; TG-triglycerides; Cholesterol HDL-high density lipoprotein cholesterol; LDL-low density lipoprotein cholesterol; eGFR-estimated glomerular filtration rate; $n$ is the number of patients; ${ }^{*}-\mathrm{p}<0.05$ between the $1^{\text {st }}$ subgroup; ${ }^{* *}-\mathrm{p}<0.05$ between the $2^{\text {nd }}$ subgroup; ${ }^{* * *}-\mathrm{p}<0.05$ between the $3^{\text {rd }}$ subgroup; ${ }^{* * * *}-\mathrm{p}$ $<0.05$ between the $4^{\text {th }}$ subgroup.

To study the effect of comorbid pathology on the functional status of the kidneys, we conducted a correlation analysis (Table 3 ), which showed that in the $1^{\text {st }}$ group of patients, the magnitude 
of GFR correlates closely with age ( $\mathrm{r}=-0.21 ; \mathrm{p}=0.03$ ), the level of systolic blood pressure $(r=-0.34 ; p=0.03)$ and the concentration of cystatin $\mathrm{C}$ of serum ( $\mathrm{r}=-0.87 ; \mathrm{p}=0.01)$. In the $2^{\text {nd }}$ group, a close connection was also established between the GFR and the level of systolic blood pressure $(r=-0.32 ; p=0.03)$ and the concentration of cystatin $C$ in the blood serum $(r=-0.86 ; p=0.03)$. In patients with hypertension and COPD (group 3), negative association of GFR with age ( $\mathrm{r}=-0.44 ; \mathrm{p}=0.03)$, heart rate $(\mathrm{r}=-0.36 ; \mathrm{p}=0.03)$, concentrations of Cholesterol ( $r=-0,20 ; p=0.04)$, HDL cholesterol ( $r=-0.27 ; p=0.03)$, LDL cholesterol $(r=-0.27 ; p=0.03)$, as well as cystatin $C$ of serum $(\mathrm{r}=-0.89 ; \mathrm{p}=0.01)$. In patients with a pronounced comorbid state (group 4), the GFR value was significantly correlated with the level of systolic blood pressure ( $\mathrm{r}=-0.33 ; \mathrm{p}=0.03)$ and the concentration of cystatin $\mathrm{C}$ in the blood serum ( $\mathrm{r}=-0.86$; $\mathrm{p}=0.01$ ) (Table 3 ).

Table 3: Comparative Clinical and Laboratory Correlation Characteristics of Surveyed Subgroups.

\begin{tabular}{|c|c|c|c|c|c|c|c|c|}
\hline \multirow{3}{*}{ Parametersofthe group } & \multicolumn{2}{|c|}{$A H+D M, n=99$} & \multicolumn{2}{|c|}{$A H+C H D, n=138$} & \multicolumn{2}{|c|}{$A H+C O P D, n=102$} & \multicolumn{2}{|c|}{$\begin{array}{c}\mathrm{AH}+\mathrm{DM}+\mathrm{CHD}+\mathrm{COPD}, \\
\mathrm{n}=44\end{array}$} \\
\hline & \multicolumn{8}{|c|}{ Estimated glomerular filtration rate, $\mathrm{ml} / \mathrm{min}$} \\
\hline & $r=$ & $\mathrm{p}=$ & $r=$ & $\mathrm{p}=$ & $r=$ & $\mathrm{p}=$ & $r=$ & $\mathrm{p}=$ \\
\hline Age, years & 0,21 & 0,03 & 0,11 & 0,19 & 0,44 & 0,03 & 0,10 & 0,48 \\
\hline BMI, kg/ $\mathrm{M}^{2}$ & 0,11 & 0,31 & 0,09 & 0,29 & 0,03 & 0,77 & 0,05 & 0,72 \\
\hline SBP, мм Hg & 0,34 & 0,03 & 0,32 & 0,03 & 0,03 & 0,77 & 0,33 & 0,03 \\
\hline DBP, мм Hg & 0,07 & 0,53 & 0,04 & 0,68 & 0,06 & 0,61 & 0,05 & 0,75 \\
\hline HR, beats/min & 0,02 & 0,82 & 0,01 & 0,90 & 0,36 & 0,03 & 0,04 & 0,80 \\
\hline Cholesterol, mmol/l & 0,04 & 0,69 & 0,05 & 0,49 & 0,20 & 0,04 & 0,04 & 0,77 \\
\hline $\mathrm{TG}, \mathrm{mmol} / \mathrm{l}$ & 0,09 & 0,37 & 0,08 & 0,35 & 0,03 & 0,78 & 0,16 & 0,26 \\
\hline HDL cholesterol, mmol/l & 0,08 & 0,41 & 0,11 & 0,19 & 0,27 & 0,01 & 0,15 & 0,31 \\
\hline LDL cholesterol, mmol/l & 0,05 & 0,58 & 0,02 & 0,74 & 0,27 & 0,01 & 0,26 & 0,86 \\
\hline Cystatin C, mg/l & 0,87 & 0,01 & 0,86 & 0,01 & 0,89 & 0,01 & 0,86 & 0,01 \\
\hline
\end{tabular}

Note: AH-arterial hypertension; DM-Diabetes mellitus; CHDcoronary heart disease; COPD-chronic obstructive pulmonary disease; BMI-body mass index; SBP-systolic blood pressure; DBP-diastolic blood pressure; HR-heart rate; Cholesterol-total cholesterol; TG-triglycerides; Cholesterol HDL-high density lipoprotein cholesterol; LDL-low density lipoprotein cholesterol; eGFR-estimated glomerular filtration rate; n-is the number of patients; p-reliability; r-correlations.

\section{Discussion}

The proposed article, which develops the theses of early detection of CKD using cystatin $\mathrm{C}$, is a continuation of the work we began to study the relationship of cystatin $\mathrm{C}$ with clinical and laboratory factors associated with a decrease in GFR $[12,13]$. Cystatin C is a protein consisting of 120 amino acid residues and belonging to the $2^{\text {nd }}$ group of the genetic family of cystatins [14]. As shown by early studies, cystatin $\mathrm{C}$ is contained in human blood plasma and excreted from the body by the kidneys [14]. It is established that the concentration of cystatin $\mathrm{C}$ is a more accurate marker of renal function than the creatinine level [14,15]. Currently, cystatin C, along with inulin, is considered the "gold standard" for determining GFR as an integral indicator of renal function, and its concentration in blood serum closely correlates with GFR $[14,15]$. According to our study, a statistically significant relationship between the concentration of cystatin C and GFR was observed in patients with comorbid pathologies. According to individual works, one of the manifestations of comorbid pathology is CKD, the wider prevalence of which is recognized by all researchers $[1,2,15]$. The adverse prognosis that distinguishes the group of comorbid pathologies with CKD is largely related to the magnitude of GFR. It should be noted that in our study, optimal renal function (C1) was detected in only $7.5 \%$ of the examined individuals with comorbid pathology (Table 1). Quite often, C2 (46.7\%) and C3 "A" (24.8\%) were observed in gradations of impaired renal function in comorbid patients (Table 1). Dragunov D.O.and authors (2016) showed that of 337 comorbid patients, the presence of CKD was noted in $43 \%$, of which $18.7 \%$ had C2 stage and 24\% C3 stage. It should be noted that the authors in this work calculated the GFR using the CKD-EPI formula [16].

In another single-stage study, it was found that the incidence of CKD among hospitalized patients of the therapeutic hospital was $47.8 \%$. Moreover, in the majority of the examined patients, C2 (18.6\%) and C3 (14.5\%) grades of CKD were detected [17]. The CKD group consisted of elderly patients, among whom women were significantly more common. This fact was confirmed in our study, i.e. the proportion of elderly people is 162 people, which accounted for $42.2 \%$ of cases (Table 1 ).

The presence of kidney damage in patients with comorbid pathology causes an additional risk of cardio and cerebrovascular events and is closely associated with the general pathogenesis of pathological processes in the vascular wall [7]. A recent study found that the combination of CHD with diabetes is accompanied by an increase in plasma concentrations of urea, as well as more severe dyslipidemia [18]. As shown in Table 3, in patients with a combination of AH and COPD, the negative association of GFR was recorded with age, heart rate, concentration of cholesterol, HDL cholesterol, LDL cholesterol, and cystatin C of serum. The noted correlation characteristics are to some extent explained by a more pronounced inflammatory syndrome [19], endothelial dysfunction, 
oxidative stress and stiffness of the vascular wall, which, in turn, contributes to the progression of the atherosclerotic process and potentiates adverse cardio-and nephrocerebral effects.

T.A. Aksenovawith authors (2018) based on a study of the pulse wave velocity (PWV) and arterial stiffness parameters in patients with comorbidity, it was demonstrated that with the combination of hypertension and COPD, systolic aortic pressure and PWV were significantly higher compared with patients with isolated hypertension and a group of people with COPD [20]. The role of increasing central BP and PWV as factors of CKD progression was summarized in our previous studies [21]. Often CKD occurs and progresses against the background of comorbid and age-related diseases, between which and a decrease in GFR there is a reliable bilateral pathogenetic relationship: the appearance of comorbid pathology aggravates the course of renal dysfunction, and vice versa, any diseases of the aging humans can be induced and contribute to the progression of CKD $[22,23]$.

Thus, in our patients with $\mathrm{AH}+\mathrm{DM}$, significantly higher concentrations of cystatin $\mathrm{C}$ were observed in blood serum compared with other subgroups (Table 2). Whereas, in the presence of pronounced comorbidity (group 4), a noticeable decrease in GFR was recorded. Apparently, this fact is also explained by the presence of CKD risk factors in this group, such as overweight and reduced levels of HDL cholesterol. However, during the correlation analysis, the presence of a close relationship of the magnitude of the GFR with the levels of BMI and cholesterol HDL was not obtained (Table 3). In our opinion, this is due to the small sample size in the group under discussion. A number of authors note that combinations of two and three nosology are most common in a doctor's practice, but in isolated cases (up to 2.7\%) one patient has a combination of 6-8 diseases simultaneously [24].

At the same time, the presence of several chronic diseases in one patient is closely related to age. Thus, in our work among the study participants, the proportion of elderly people was $42.2 \%$ (Table 1). According to K. Barnett and authors [25], the frequency of comorbid pathology was $23 \%$, and the majority of patients were over 65 years of age [18]. The facts of increasing the concentration of cystatin C, as well as the presence of a close relationship between the levels of systolic blood pressure, cholesterol, HDL cholesterol, LDL cholesterol and GFR in comorbid patients examined by us, make a definite contribution to the CKD formation. Conclusion.

In patients with comorbid pathology, the frequency of occurrence of a decrease in GFR of C2 gradation was 46.7\%, and C3 "A"-24.8\%. In general, severe decline in renal function (grades C4 and C5) was observed in $11.7 \%$ of patients and was characteristic for more women than men. Laboratory predictors associated with decreased renal function were the presence of hypercholesterolemia, hypertriglyceridemia and elevated levels of low-density lipoprotein cholesterol [26]. In individuals with comorbid pathology, the incidence and severity of chronic kidney disease are influenced by age, body mass index, systolic blood pressure, total cholesterol, low-density lipoprotein cholesterol, and cystatin $\mathrm{C}$ of plasma. At the same time, the combination of arterial hypertension with diabetes mellitus type 2 and severe comorbid pathology contributes to a more noticeable reduction in renal function.

\section{Acknowledgement}

None.

\section{Conflict of Interest}

The authors declare the absence of overt and potential conflicts of interest related to the publication of this article.

\section{References}

1. Moiseev VC, Mukhin NA, Smirnov AV (2014) Cardiovascular risk and chronic kidney disease: cardio-nephroprotection strategies. Russian Journal of Cardiology 8: 7-37.

2. Whittaker CF, Miklich MA, Patel RS, Fink JC (2018) Medication safety principles and practice in CKD. Clin J Am Soc Nephrol 13(11): 17381746

3. Feinstein AR (1970) The pre therapeutic classification of comorbidity in chronic disease. J Chronic Dis 23(7) 455-468.

4. Zhang M, Holman CD, Price SD (2009) Comorbidity and repeat admission to hospital for adverse drug reactions in older adults: retrospective cohort study. BMJ 338: a2752.

5. Chukayeva II, SamorodskayaI V, Larina VN (2018) The prevalence of multimorbidity: discussion about the terminology, registration and its effect on patient's health care utilization. Ter Arkh 8(90): 125-130.

6. Drapkina OM, Shutov AM, Efremova EV (2019) Comorbidity, multimorbidity, dual diagnosis - synonyms or different terms? Cardiovascular Therapy and Prevention 18(2): 65-69.

7. Barbarash OL, Zhidkova II, Shibanova IA (2019) The impact of comorbidities and age on the nosocomial outcomes of patients undergoing coronary artery bypass grafting. Cardiovascular Therapy and Prevention 18(2): 58-64.

8. Oganov RG, Simanenkov VI, Bakulin IG (2019) Comorbiditi es inclinical practice. Algorithms for diagnostics and treatment. Cardiovascular Therapy and Prevention 18(1): 5-66.

9. Hirlekar G, Jonsson M, Karlsson T (2018) Comorbidity and survival in out-of-hospital cardiac arrest. Resuscitation 133: 118-123.

10. Taylor DM, Fraser SD, Bradley JA (2017) A systematic review of the prevalence and associations of limited health literacy in CKD. Clin J Am Soc Nephrol 12(7): 1070-1084.

11. Hoek FJ, Kemperman FA, Krediet RT (2003) A comparison between cystatin C, plasma creatinine and the Cockcroft and Gault formula for the estimation of glomerular filtration rate. Nephrol Dial Transplant 18(10): 2024-2031.

12. Tsygankov VA, Zharkova SL (2009) Classification and systematization of the able-bodied population in age groups. The Journal Omsk Scientific Bulletin 4(79): 67-70.

13. Murkamilov IT, Sabirov IS, Fomin VV, Murkamilova JA, Aitbaev KA, et al. (2018) Evaluation of nephrocerebral risk using cystatin C in patients with chronic kidney disease. Zh Nevrol Psikhiatr Im S S Korsakova 118(9): 10-16.

14. Murkamilov IT, Fomin VV, Aitbaev KA (2018) Blood plasma cystatin $\mathrm{C}$ level and its relationship with the augmentation index and central arterial pressure in therapeutic patients. Clinical nephrology (3): 31-40.

15. Filler G, Bökenkamp A, Hofmann W, Le Bricon T, Martínez-Brú C, et al. (2005) Cystatin C as a marker of GFR-history, indications, and future research. Clin Biochem 38(1): 1-8.

16. Shlipak MG, Matsushita K, Ärnlöv J, Inker LA, Katz R, et al. (2013) Cystatin $\mathrm{C}$ versus creatinine in determining risk based on kidney function. $\mathrm{N}$ Engl J Med 369(10): 932-943. 
17. Dragunov DO, Sokolova AV, Latyshev TV, Arutyunov GP (2016) Prevalence of chronic kidney disease in comorbid patients. Klinicheskayanefrologiya (3-4): 49-52.

18. Pigareva Yu A, Avdoshina SV, Dmitrova TB (2012) The prevalence of chronic kidney disease among patients of the therapeutic hospital. Clinical nephrology 3: 4-9.

19. Akhmedova EB, Mardanov BU, Mamedov MN (2017) Influence of somatic comorbidity on the course of coronary heart disease. Russian Journal of Cardiology (9): 55-59.

20. Smirnova EN, Smirnova LE, Egorova EN (2015) Immunological characteristics of patients with comorbid chronic obstructive pulmonary disease and arterial hypertension. The Transbaikalian medical bulletin 3: 95-101.

21. Aksenova TA, Gorbunov VV, Tsarenok SY (2018) Pulse wave velocity and other indicators of arterial stiffness in hypertension comorbidity and chronic obstructive pulmonary disease. Ter arkh 3(90): 10-15.
22. Murkamilov IT, Sabirov IS, Aitbaev KA, Fomin VV, Murkamilova ZA, et al. (2018) Renal dysfunction and indicators of arterial hormity in persons of elderly and senile age. Adv Gerontol 31(4): 549-555.

23. Melikhova SP, Shevcova VI, Zujkova AA, Kotova Yu A (2018) Studying of comorbid pathology at the 2 types diabetes as the complication of the metabolic syndrome. The Russian Archives of Internal Medicine 8(5): 366-371.

24. Vishnevskii KA, Belyaev AE, Mironenko AN (2015) Polymorbidity as a predictor of mortality for hemodialysis patients. Nephrology (SaintPetersburg) 19(4): 89-94

25. Vyortkin AL (2015) Comorbidity: history, modern presentation, prevention and treatment. Cardiovascular Therapy and Prevention 14(2): 74-79.

26. Barnett K, Mercer SW, Norbury M, Watt G, Wyke S, et al. (2012) Epidemiology of multimorbidity and implications for health care, research, and medical education: a cross-sectional study. Lancet 380(9836): 37-43. 